\title{
Experience with Crystals at Fermilab Accelerators
}

\author{
V. D. Shiltsev* \\ Fermi National Accelerator Laboratory, MS 312, \\ Batavia, IL 60510, USA \\ *E-mail: shiltsev@fnal.gov
}

\begin{abstract}
Crystals were used at Fermilab accelerators for slow extraction and halo collimation in the Tevatron collider, and for channeling radiation generation experiments at the FAST electron linac facility. Here we overview past experience and major outcomes of these studies and discuss opportunities for new crystal acceleration R\&D program.
\end{abstract}

Keywords: Colliders; accelerators; crystals; collimation; halo.

\section{Slow extraction of the Tevatron beams}

Strong interplanar fields in crystals $O(10 \mathrm{~V} / \AA)$ can be effectively used for various manipulations over charged particles with even the highest energies ${ }^{1}$, such as $1 \mathrm{TeV}$ protons in the Tevatron ${ }^{2}$ or $6.5 \mathrm{TeV}$ ones in the $\mathrm{LHC}^{3}$.

Following the 1980's exploratory experiments with crystals in secondary particle beams at Fermilab ${ }^{5}$, a series of slow extraction studies at the Tevatron collider, led by R.Carrigan and organized under the umbrella of the Fermilab Experiment E853, had taken place in the $1990 \mathrm{~s}^{6,7}$. Detailed technical description, scientific results and historical background can be found in ${ }^{8,9}$.

Luminosity driven channeling extraction has been observed for the first time using a $900 \mathrm{GeV}$ circulating proton beam (the highest energy at that time) at the superconducting Fermilab Tevatron ${ }^{8}$ - see Fig.1 a). The extraction efficiency was found to be about $30 \%$. A $150 \mathrm{kHz}$ beam was obtained during luminosity driven extraction with a tolerable background rate at the collider experiments. A $900 \mathrm{kHz}$ beam was obtained when the background limits were doubled.

In the follow-up E853 development ${ }^{9}$, the beam extraction efficiency was about $25 \%$. Studies of time dependent effects found that the turn-to-turn structure was governed mainly by accelerator beam dynamics. Based on the results of the E853 experiment, feasibility of a parasitic 5-10 MHz proton beam from the Tevatron collider was established.

The extracted proton flux depended strongly on the crystal alignment with typical rms width of $\pm 2 \mu \mathrm{rad}$ - see Figs. $1 \mathrm{~b}$ ) and $1 \mathrm{c}$ ) - and on the source of the particle's diffusions: e.g., it scaled quadratically with the power of the tranvesrse dipole noise heating and linearly with the proton-antiproton luminosity in the range $(0.1-1) \cdot 10^{30} \mathrm{~cm}^{-2} \mathrm{~s}^{-1}$. 

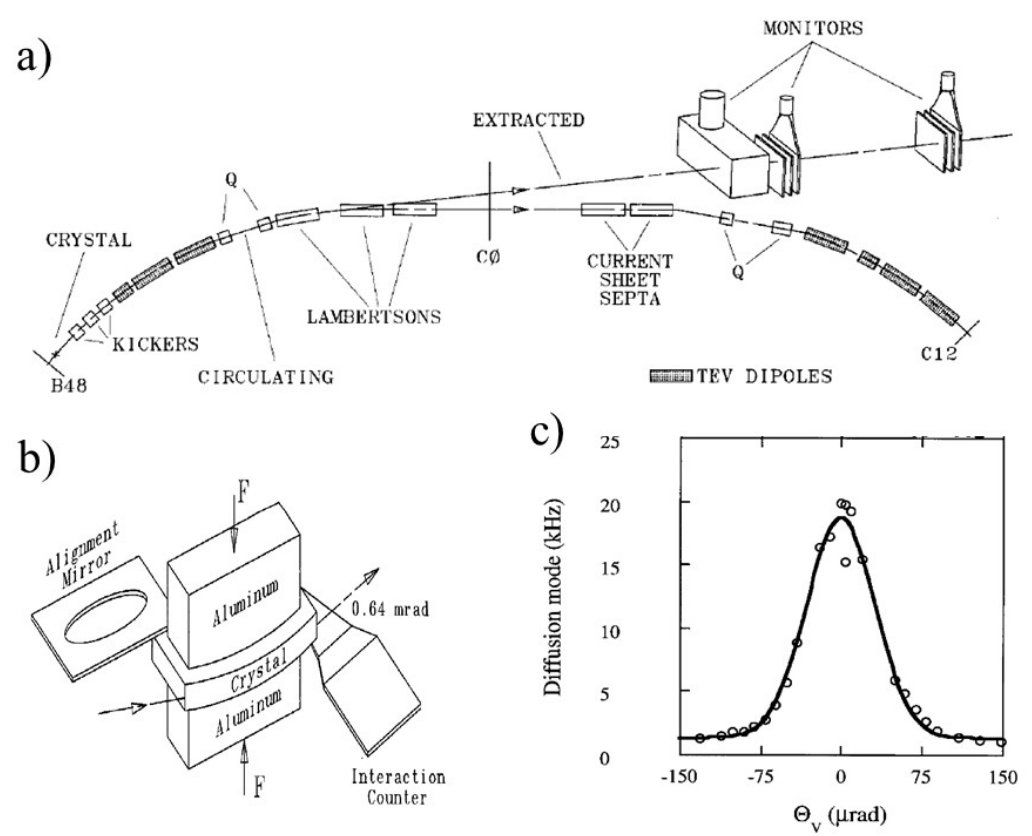

Fig. 1. Crystal assisted proton extraction from the Tevatron: a) schematic of the E853 experiment; b) $39 \mathrm{~mm}$ long, $3 \mathrm{~mm}$ high, $9 \mathrm{~mm}$ wide bent Si crystal cut along (111) plane; c) dependence of the extracted proton flux on angular alignment of the crystal (adapted from ${ }^{8,9}$ ).

\section{Halo collimation by bent crystals in the Tevatron}

The Tevatron Run II (2001-2011) ${ }^{2}$ employed a two-stage collimation system in which a primary collimator is used to increase the betatron oscillation amplitudes of the halo particles, thereby increasing their impact parameters on secondary collimators ${ }^{10}$. A bent crystal can coherently direct channeled halo particles deeper into a nearby secondary absorber, thus, reducing beam losses in critical locations around the Tevatron ring and the radiation load to the downstream superconducting magnets ${ }^{11}$. There are several processes which can take place during the passage of protons through the crystals: a) amorphous scattering of the primary beam; b) channeling; c) dechanneling due to scattering in the bulk of the crystal; d) volume reflection off the bent planes; and e) volume capture of initailly unchanneled particles into the channeling regime after scattering inside the crystal. Particle can be captured in the channeling regime, oscillating between two neighboring planes if it enters within crystals angular acceptance (critical angle) of:

$$
\theta_{c}=\sqrt{\frac{2 U_{0}}{p c}} .
$$

where $p$ is the particle momentum and $U_{0}$ is the crystals planar potential well depth. For $980 \mathrm{GeV} / \mathrm{c}$ protons in the Tevatron, the critical angle for (110) plane 
of a silicon crystal is about $7 \mu \mathrm{rad}$. If the particle momentum is not within the critical angle but has a tangency point with the bent planes within the crystal volume, almost all particles are deflected to the opposite direction with respect to the crystal bending - the effect is called the volume reflection (VR) which has very wide angular acceptance equal to the crystal bend angle of the order of hundreds of microradians (vs several microradians of the channeling acceptance). The drawback of the volume reflection regime is that the deflection angle is small, approximately $(1.5-2) \times \theta_{c}$. However, this can be overcome by using a sequence of several precisely aligned bent crystals, so that the total deflection angle is proportionally larger.

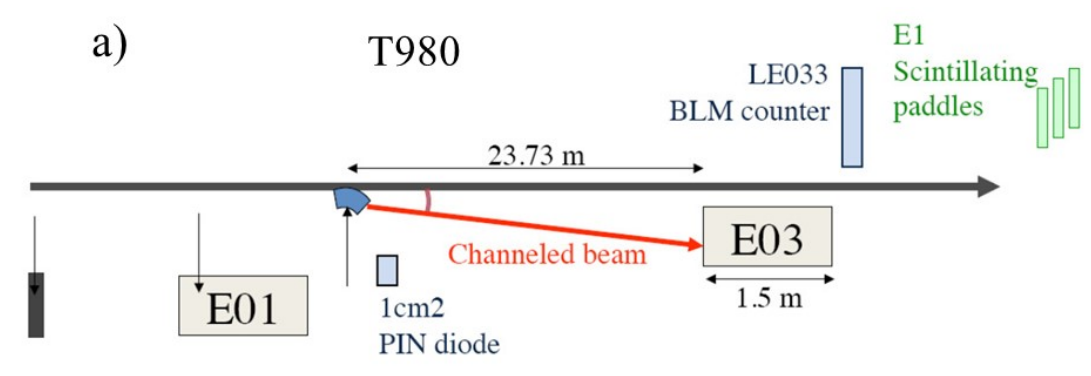

b)

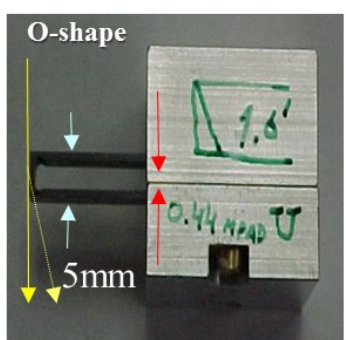

c)

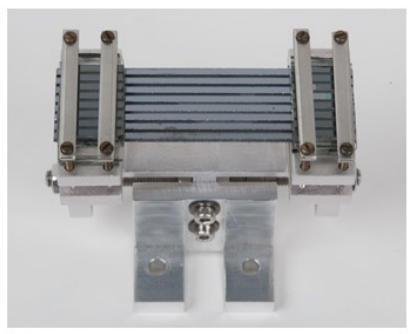

d)

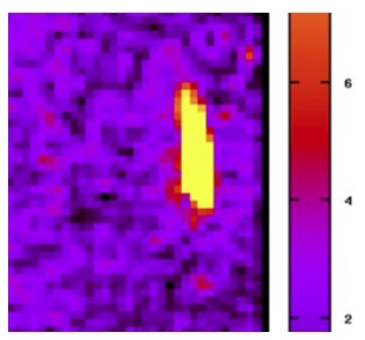

Fig. 2. Bent crystal collimation in the Tevatron: a) schematic of the T980 setup; b) O-shaped 5$\mathrm{mm}$ silicon crystal with a bending angle of $0.44 \mathrm{mrad}$; c) eight strip crystal assembly for consecutive volume reflections; d) image of a pixel detector near E03 collimator showing the channeled beam profile (adapted from ${ }^{2,11}$ ).

In the Tevatron beam crystal collimation experiment T980 ${ }^{11}$ both single crystals (for vertical and horizontal deflection) and multi-strip crystal assemblies (for vertical multiple VR) have been used. The bent crystal collimation of circulating beams is very different from that of extracted beams because of smaller initial impact parameter (the depth of the particle penetration at the first interaction with the crystal) and the possibility of interplay of different effects. In an accelerator such as the Tevatron several phenomena determine the impact parameter: diffusion due to scattering on the residual gas and noise in magnetic field $\sim 4 \mathrm{~nm} / \operatorname{turn}^{1 / 2}$; the 
RF noise results in the diffusion rate of $\sim 12 \mathrm{~nm} / \operatorname{turn}^{1 / 2}$ (hor.) and $\sim 4 \mathrm{~nm} / \operatorname{turn}^{1 / 2}$ (vert.); beam diffusion due to beam-beam or other nonlinear effects can produce up to $\sim(10-40) \mathrm{nm} / \operatorname{turn}^{1 / 2}$. Interactions with amorphous targets lead to the diffusion rate $\sim 200 \mu \mathrm{m} / \operatorname{turn}^{1 / 2}$ for a $5 \mathrm{~mm}$ length of amorphous silicon, and about $\sim 4$ $1200 \mu \mathrm{m} /$ turn $^{1 / 2}$ for a $5 \mathrm{~mm}$ tungsten primary target. Also, of importance transverse orbit oscillations with amplitude of about $20 \mu \mathrm{m}$ and frequencies of $15 \mathrm{~Hz}$ and the $35 \mathrm{~Hz}$ (1300 turns) synchrotron motion of particles near the boundary of the RF bucket with amplitudes of some $1 \mathrm{~mm}$ (hor) and some $70 \mu \mathrm{m}$ (vert). The resulting impact parameters are estimated to be of the order of $0.2-1 \mu \mathrm{m}$ for transverse halo particles and 10-30 $\mu \mathrm{m}$ for the particles in the abort gaps which have leaked out of the RF buckets. All that makes the properties of the surface of the crystal, its roughness and/or the miscut angle (rather than the bulk of the crystal) pivotal for the halo collimation.

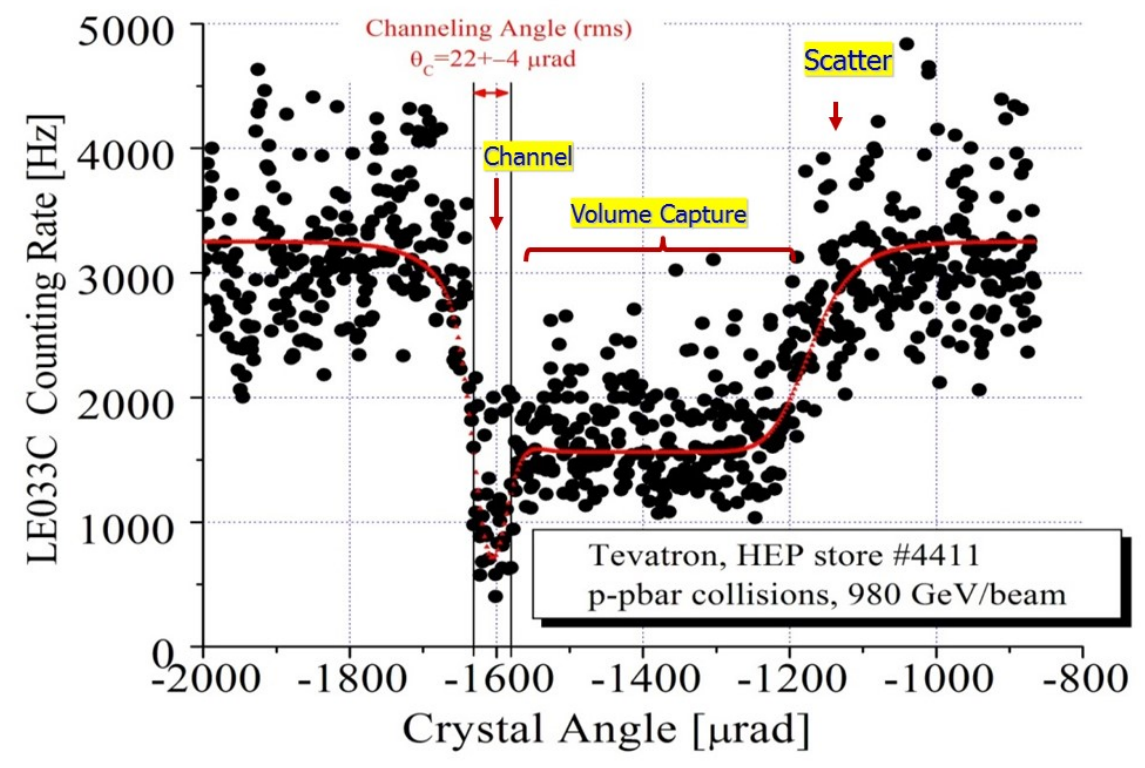

Fig. 3. The scan of the near crystal radiation counter signal (interaction probability of $980 \mathrm{GeV}$ protons with $\mathrm{Si}$ ) vs crystal alignment angle (adapted from ${ }^{2}$ ).

Figure 2 shows a schematic of the T-980 experimental layout, some critical hardware and results. During normal Tevatron operations, a 5-mm tungsten target scatters the proton beam halo into a $1.5-\mathrm{m}$ long stainless steel secondary collimator E03, $50 \mathrm{~m}$ downstream of the target. For the bent crystal experiments, a goniometer containing single or multi-strip bent crystals is installed $23.7 \mathrm{~m}$ upstream of the 
Table 1. Main parameters of the FAST electron beam.

\begin{tabular}{|c|c|c|c|}
\hline Parameter & $\begin{array}{c}\text { FAST } \\
2019\end{array}$ & ILC specs. & Comments \\
\hline $\begin{array}{c}\text { Beam energy: max. } \\
\text { low-energy area } \\
\text { electron gun }\end{array}$ & $\begin{array}{c}301 \mathrm{MeV} \\
20-50 \mathrm{MeV} \\
4-5 \mathrm{MeV}\end{array}$ & $300 \mathrm{MeV}$ & $\begin{array}{l}100 \mathrm{MeV} \text { for IOTA } \\
\text { typical } 34-43 \mathrm{MeV} \\
\text { typical } 4.5 \mathrm{MeV}\end{array}$ \\
\hline Bunch charge & $0.1-3.2 \mathrm{nC}$ & $3.2 \mathrm{nC}$ & $\begin{array}{l}\text { typical } 0.5 \mathrm{nC} \text {, depends } \\
\text { on number of bunches }\end{array}$ \\
\hline Bunches per pulse & $1-1000$ & 3000 & typical $100,3 \mathrm{MHz}$ rate \\
\hline Pulse length & upto $1 \mathrm{~ms}$ & $1.0 \mathrm{~ms}$ & typical $0.01-0.2 \mathrm{~ms}$ \\
\hline Pulse rep.rate & $1 \mathrm{~Hz}$ & $5 \mathrm{~Hz}$ & \\
\hline Transv. emittance (n, rms) & $1-5 \mu \mathrm{m}$ & $5 \mu \mathrm{m}$ & grows with intensity \\
\hline Bunch length (rms) & $1.2-2.4 \mathrm{~mm}$ & $1 \mathrm{~mm}$ & without compression \\
\hline
\end{tabular}

E03 collimator. Scintillation counter telescopes detect secondary particles from protons interacting with the target and E03 collimator. An ionization chamber (beam loss monitor LE033) also detects secondary particles scattered from E03. A PIN diode telescope detects the secondaries scattered from the bent crystal. Under the above configuration, channeled beam is signaled by a reduction of the rate in the PIN telescope (channel LE033C) - as shown in Fig.3. Depending on the angle between the incident particle's orbit and the Si crystal plane, observed are channeling dip with a width of $22 \pm 4 \mu \mathrm{rad}$ ( $\mathrm{rms}$ ), volume reflection plateau and amorphous scattering. The width of the channeling dip is a convolution of the beam divergence, the channeling critical angle, multi-pass channeling effects and possible crystal distortions. At the bottom of the dip, the LE033C signal is about $20 \%$ of the signal at a random angular setting. This depth is a measure of the channeling efficiency of $80 \%$.

The crystal collimation had been used during many collider stores in 2010's with peak luminosities upto $400 \cdot 10^{30} \mathrm{~cm}^{-2} \mathrm{~s}^{-1}$. Since 2009 , the system had pushpull crystal goniometers in both planes and a two-plane crystal collimation has been attempted for the first time. High-resolution pixel telescope was installed in front the downstream collimator and allowed to measure channeled and volume reflected beam profiles at those location - see Fig.2 d). Also, observed were multiple-volume reflected protons (off an 8-crystal assembly in which each strip coherently adds a $8 \mu \mathrm{rad}$ bend to the same proton) and a two-fold reduction of beam losses around the ring due to crystal channeling, specifically in the CDF and D0 collider detector regions.

\section{Crystal channeling radiation experiment at FAST}

Channeling radiation (CR) can be generated when charged particles such as electrons or positrons pass through a single crystal parallel to a crystal plane or axis. The main advantage of the $\mathrm{CR}$ is to produce quasi-monochromatic high energy X-rays using a low energy electron beam below $100 \mathrm{MeV}$ (compare with the synchrotron radiation, currently the main X-ray source that requires a few $\mathrm{GeV}$ electron 
beams to generate X-rays of tens of $\mathrm{keV}$ ).

Recently constructed Fermilab Accelerators Science and Technology (FAST) facility $^{12}$ hosted series of studies aimed to produce high brightness X-rays using a low-emittance $50 \mathrm{MeV}$ electron beam, and to demonstrate that $\mathrm{CR}$ can be used as a compact high-brightness X-rays source ${ }^{13,14}$. The FAST injector ${ }^{15}$ (see main parameters in Table 1) which consists of a CsTe photocathode located in a $1+1 / 2$-cell RF gun followed by two L-band $(1.3 \mathrm{GHz})$ superconducting accelerating structures can generate a low emittance electron beam. The electron energy can reach up to $50 \mathrm{MeV}$ downstream of the last superconducting cavity. A $160 \mu \mathrm{m}$ thick diamond single crystal (with some $1 \mathrm{mrad}$ critical angle for $43 \mathrm{MeV}$ electrons) was installed and oriented so that the electron beam propagates parallel to the (110) plane of the crystal - see Fig.4.

a)

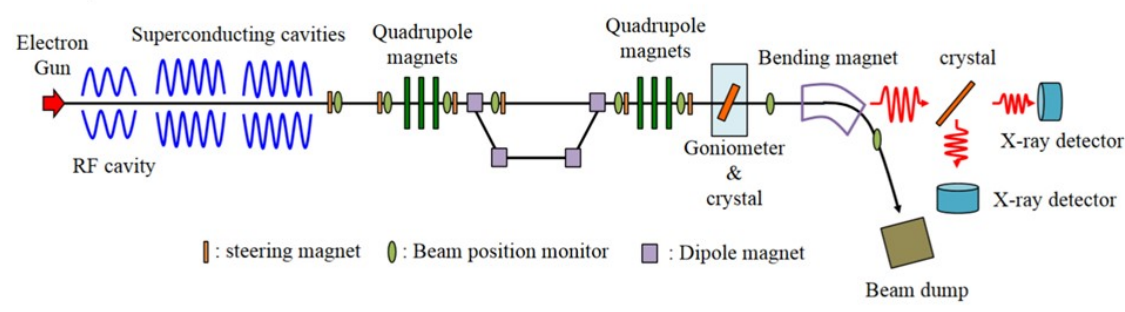

b)

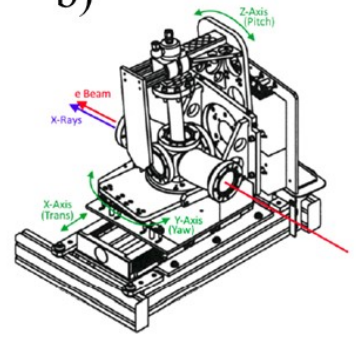

c)

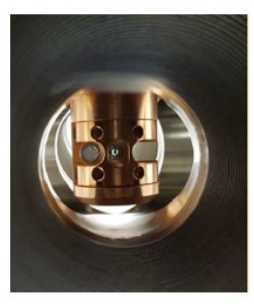

d)

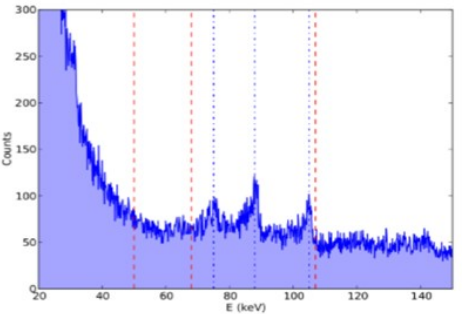

Fig. 4. Crystal channeling radiation experiment at FAST: a) schematic view of the experiment; b) crystal goniometer assembly; c) crystal goniometer assembly installed in low energy electron beamline; d) energy spectrum of emitted X-rays (adapted from ${ }^{16,17}$ ).

Many channeling spectra were summed together for 30-fC/pulse electron beam in Fig.4d) indicating three peaks over the bremstrahlung (BS) background spectrum at $75 \mathrm{keV}, 87 \mathrm{KeV}$ and $108 \mathrm{keV}^{16}$. Originally, the peaks were expected at $51 \mathrm{keV}$, $67 \mathrm{keV}$ with much higher signal above the BS background ${ }^{17}$ and the discrepancy might be explained by the effects of nonlinear detector performance in the radiation environment, crystal morphological changes due to aging, or the electron beamline 
energy measurement offset.

(a)

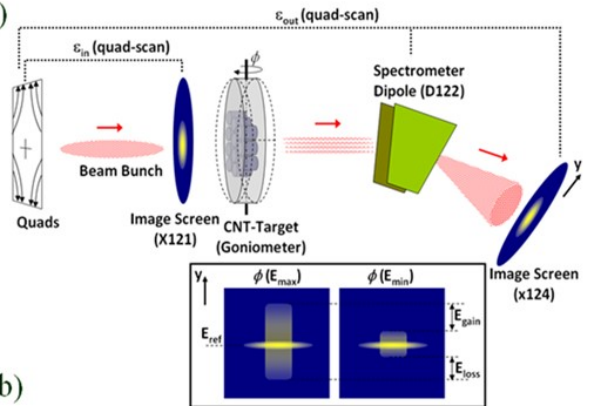

(c)

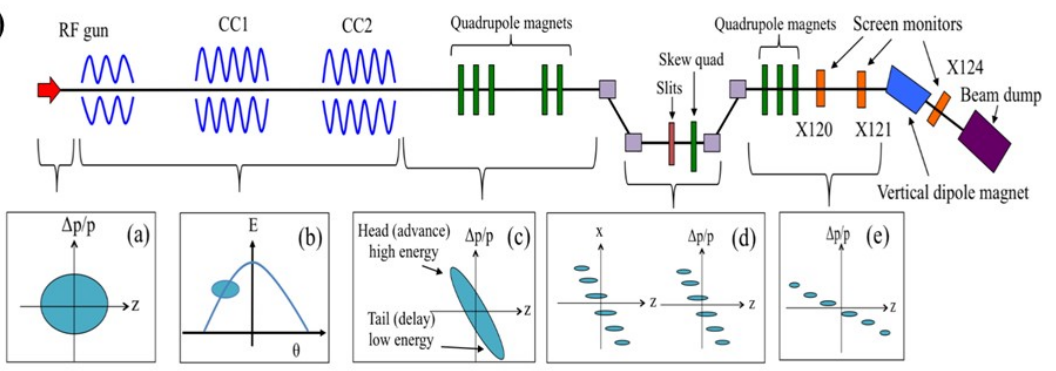

Fig. 5. Proof-of-principle CNT acceleration experiment proposal (see text): a) focusing premodulated electron beam on a CNT plate, b) simulated beam image on a screen after the vertical bending spectrometer magnet with(left) and without(right) proper CNT alignment; c) concept of the $\mathrm{THz}$ microbunching of the FAST electron bunch using vertical slit-mask in the chicane (adapted from ${ }^{20,21}$ ).

\section{Possibilities for CNT acceleration tests at FAST}

Initial outlook for ultimate future energy frontier collider facility with beam energies 20-100 times the LHC energy indicates promising potential of a compact $1 \mathrm{PeV}$ linear crystal collider based on acceleration of muons (instead of electrons or hadrons) in super-dense plasma of crystals was ${ }^{18}$. Nanostructures, such as carbon nanotubes (CNTs) can also be used offering a number of advantages over crystals for a proof-ofprinciple experiment (wider channels and weaker dechanneling; possibility to accept broader beams using nanotube ropes, easier 3D control of beam bending over greater lengths $)^{19}$. Simulations of the proposal of such a study at FAST ${ }^{20}$ assume excitation of wakefields in the CNT plasma by short intense pre-modulated electron bunch - see Fig.5. The beam density modulation (microbunching) is needed for resonant excitation of the wakefields at the CNT plasma wavelength $\lambda_{p} \sim 100 \mu \mathrm{m}$ and can be arranged via, e.g., the use of a narrow vertical slit mask in the chicane section of the low-energy FAST beamline, as depicted in Fig. 5 c) ${ }^{21}$. The effect 
of $\sim 300 \mathrm{keV}$ CNT acceleration will manifest itself as widening of the vertical image size on the beam image screen after the spectrometer magnet .

Another possibility of using $300 \mathrm{MeV}$ electron beam at FAST for effective muon production was discussed in ${ }^{22}$ where efficiency of $e \rightarrow \mu$ conversion was estimated to be $O\left(10^{-8}\right)$. FAST beams can be used for related studies of the electron beam filamentation (Weibel instability) in solid density plasmas, muon detection, calibration of theoretical models and integration of future experiments on acceleration in crystals at lager facilities, such as, e.g., FACET-II.

\section{Conclusions}

Fermilab has a long history of research and operational use of crystals in high energy accelerators starting with employment of them in extracted and secondary beamlines in 1980's, followed by the pioneering E853 experiment on crystal assisted proton extraction by R.Carrigan, et al. during the Tevatron Collider Run I in 1990's and the T980 crystal collimation studies in 2005-2011 by N.Mokhov et al.. Recently constructed FAST 50-300 MeV electron linac hosted crystal channeling radiation studies and offers opportunities for various experiments towards proof-of-principle studies of acceleration in crystals and nanostructures. Significant past experience and available hardware can be very helpful for future exploration toward pre-FACET II crystal acceleration experiment (integration and tests of detectors); particle channeling in CNTs; muon production and capture. Together with other possible experiments at FACET-II at SLAC, BELLA at LBNL and AWAKE at CERN, with high energy high- $Z$ ions available at RHIC or LHC or with selfmodulated electron beams in the SASE FEL facilities, like, e.g. the LCLS-I and -II at SLAC , these studies at FAST can provide new insights into the feasibility of the concept of beam acceleration in solid-state plasma of crystals or nanostructures like CNTs, the promise of ultra-high accelerating gradients $O(1-10) \mathrm{TeV} / \mathrm{m}$ in continuous focusing channels and, thus, prospects of the method for future high energy physics colliders.

\section{Acknowledgments}

Some materials of this summary were also presented at the International Particle Accelerator Conference (IPAC'2010, May 23-28, 2010, Kyoto, Japan) ${ }^{?}$ and in Refs. ${ }^{2,10,11}$. I greatly appreciate years of fruitful collaboration on the subjects of this presentation with G.Annala, R.Carrigan, A.Drozhdin, T.Johnson, A.Legan,N. Mokhov, T.Sen, R.Reilly, D.Still, R.Tesarek, J. Thangaraj, and J.Zagel (FNAL), R.Assmann, V.Previtali, S.Redaelli, and W.Scandale (CERN), Y.Chesnokov and I.Yazynin (IHEP, Protvino, Russia), V.Guidi (INFN-Ferrara, Italy), P.Piot and Y.M.Shin (NIU), Y.Ivanov (PNPI, Russia). Special thanks to Dean Still and Nikolai Mokhov for useful discussions and help with finding the materials and photos reproduced in this article. 
Fermi National Accelerator Laboratory is operated by Fermi Research Alliance, LLC under Contract No. DE-AC02-07CH11359 with the United States Department of Energy.

\section{References}

1. V.Biryukov, Y.Chesnokov, V.Kotov, Crystal channeling and its application at highenergy accelerators (Springer, 2013).

2. V.Lebedev, V.Shiltsev (eds.), Accelerator physics at the Tevatron collider (Springer, 2014).

3. W.Scandale, et al., Observation of channeling for $6500 \mathrm{GeV} / \mathrm{c}$ protons in the crystal assisted collimation setup for LHC, Phys. Lett. B 758, 129 (2016).

4. V.Shiltsev, High-energy particle colliders: past 20 years, next 20 years, and beyond. Physics-Uspekhi 55, (10), 965 (2012).

5. S.Baker, et al., Deflection of charged particles in the hundred $\mathrm{GeV}$ regime using channeling in bent single crystals, Phys. Lett. B 137 (1-2), 129 (1984).

6. R.Carrigan, T.Toohig, E.Tsyganov, Beam extraction from $\mathrm{TeV}$ accelerators using channeling in bent crystals, Nucl. Instr. Meth. B 48(1-4), 167 (1990).

7. R.Carrigan, et al., Extraction from TeV-range accelerators using bent crystal channeling, Nucl. Instr. Meth. B 90(1-4), 194 (1994).

8. R.Carrigan, et al., First observation of luminosity-driven extraction using channeling with a bent crystal, Phys. Rev. ST-AB 1(2), 022801 (1998).

9. R.Carrigan, et al., Beam extraction studies at $900 \mathrm{GeV}$ using a channeling crystal, Phys. Rev. ST-AB 5(4), 043501 (2002).

10. N.Mokhov, et al., Tevatron beam halo collimation system: design, operational experience and new methods, Journ.Instr. 6(08), T08005 (2011).

11. N.Mokhov, et al., Crystal collimation studies at the Tevatron (T-980), Int. Journ. Mod. Phys. A 25, 98 (2010).

12. S.Antipov, et al., IOTA (Integrable Optics Test Accelerator): facility and experimental beam physics program, JINST 12, T03002 (2017).

13. T. Sen, C.Lynn, Spectral brilliance of channeling radiation at the ASTA photoinjector, Int. J. Mod. Phys. A. 29, 1450179 (2014).

14. D. Mihalcea, et al., Channeling Radiation Experiment at Fermilab ASTA, in Proc. IPAC2015 (Richmond, VA, USA, 2015), pp.95-98.

15. D. Broemmelsiek, et al., Record high-gradient SRF beam acceleration at Fermilab, New Journ. Phys. 20(11), 113018 (2018).

16. A.Halavanau, et al., Commissioning and First Results From Channeling Radiation At FAST, arXiv:1612.07358 (2016).

17. J.Hyun, P.Piot,T.Sen, Optics and bremsstrahlung estimates for channeling radiation experiments at FAST, arXiv:1802.06113 (2018).

18. V.Shiltsev, High-energy particle colliders: past 20 years, next 20 years, and beyond, Physics-Uspekhi 55(10), 965 (2012).

19. Y.M.Shin, D.Still, V.Shiltsev, X-ray driven channeling acceleration in crystals and carbon nanotubes, Phys. Plasmas 20(12), 123106 (2013).

20. Y.M.Shin, A.Lumpkin, R.Thurman-Keup. TeV/m nano-accelerator: Investigation on feasibility of CNT-channeling acceleration at Fermilab, Nucl. Instr. Meth. B $\mathbf{3 5 5}$, 94 (2015).

21. J.Hyun, et al., Micro-bunching for generating tunable narrow-band $\mathrm{THz}$ radiation at the FAST photoinjector, arXiv:1808.07846 (2018).

22. V.Shiltsev, S.Striganov, presented at the rth Annual IOTA/FAST Collaboration 
Meeting and Workshop on High Intensity Beams in rings, June 10-12, 2019, Fermi$l a b ;$ https://indico.fnal.gov/event/20279/

23. V.Shiltsev, et al., Channeling Radiation Experiment at Fermilab ASTA, in Proc. 1st Int. Part. Accel. Conf. (IPAC'2010) (May 23-28, 2010, Kyoto, Japan), pp.1242-1245. 